\title{
Pulsed Sound Waves in a Compressible Fluid
}

\author{
Pierre Hillion \\ Institut Henri Poincaré, Paris, France \\ E-mail: pierre.hillion@wanadoo.fr \\ Received July 22, 2011; revised August 23, 2011; accepted August 30, 2011
}

\begin{abstract}
The propagation along $\mathrm{Oz}$ of pulsed sound waves made of sequences of elementary unit pulses $U(\sin \tau)$ where $U$ is the unit step function and $\tau=k z-\omega t$ is analyzed using the expansion of $U(\sin \tau)$ and of the Dirac distribution $\delta(\sin \tau)$ in terms of $\tau-n \pi$ where $\mathrm{n}$ is an integer. Their properties and how these pulsed sound waves could be generated are discussed.
\end{abstract}

Keywords: Sound Wave, Unit Step Function, Travelling Pulses, Compressible Fluid

\section{Introduction}

In a compressible fluid, waves of compression with small amplitudes, sound waves, can propagate causing alternate compression and rarefaction at each point of the fluid. These travelling perturbations are periodic in opposite to noise, characterized as being aperiodic, that is having a non repetitive pattern. Pulsed sound waves are widely used in different fields such as medicine [1], underwater detection [2] or non-destructive evaluation [3].

Sound waves are generally described by a Fourier series of harmonic plane waves [4]. Here, we consider instead a sequence of elementary unit pulses. They come from the expansion [5] of the function $U(\sin \tau)$ in which $U$ is the unit step function and $\tau=k z-\omega t$, (assuming propagation along oz) in terms of $\tau-n \pi$ where $\mathrm{n}$ is an integer. A similar expansion exists for the Dirac distribution $\delta(\sin \tau)$. We discuss the properties of these pulsed sound waves and how they could be generated.

\section{Isentropic Compressible Fluid}

For an isentropic fluid in which viscosity and thermal conductivity can be neglected, the equations of motion are [4] with the pression, density, velocity $p, \rho$

$$
\begin{gathered}
\partial_{t} \rho+\boldsymbol{v} \cdot \nabla \rho+\rho \nabla \cdot \boldsymbol{v}=0 \\
\partial_{t} \boldsymbol{v}+(\boldsymbol{v} \cdot \nabla) \boldsymbol{v}+1 / \rho \nabla \rho=0,
\end{gathered}
$$

A sound wave is generated by a small disturbance of the fluid so that

$$
\begin{gathered}
|\boldsymbol{v}| \ll c=\left(\partial_{\rho} p\right)_{0}, p=p_{0}+p^{\prime}, \rho=\rho_{0}+\rho^{\prime}, \\
p^{\prime} \ll p_{0}, \rho^{\prime} \ll \rho_{0}
\end{gathered}
$$

$p_{0}, \rho_{0}$ are the pression and density in absence of disturbances and $c$ the velocity of sound.

Taking into account $(2),(\boldsymbol{v} \cdot \nabla) \boldsymbol{v}$ in $(1 \mathrm{~b})$ can be neglected, as well as the quantities of second order so that Equations $(1 \mathrm{a}, 1 \mathrm{~b})$ reduce to

$$
\begin{aligned}
& \partial_{t} \rho^{\prime}+\rho_{0} \nabla \cdot \boldsymbol{v}=0 \\
& \partial_{t} \boldsymbol{v}+\nabla p^{\prime} / \rho_{0}=0
\end{aligned}
$$

with

$$
p^{\prime}=c^{2} \rho^{\prime}
$$

Substituting (4) into (3a) gives

$$
\partial_{t} p^{\prime}+\rho_{0} c^{2} \nabla \cdot v=0
$$

and we get from (3b) with $\boldsymbol{v}=\nabla \phi$

$$
p^{\prime}=-\rho_{0} \partial_{t} \phi
$$

We then obtain from (5) the wave equation

$$
\Delta \phi-c^{-2} \partial_{t}^{2} \phi=0
$$

To sum up, once $p^{\prime}$ known from (6), (7), we get for the density and the velocity

$$
\rho^{\prime}=c^{-2} p^{\prime}, v=p^{\prime} / \rho_{0} c
$$

while for the density of energy $E$ and momentum $\boldsymbol{j}$ of the sound wave, it comes [4]

$$
\begin{gathered}
E=\frac{1}{2} \rho_{0} v^{2}+\frac{1}{2} c^{2} \rho^{\prime} / \rho_{0} \\
\boldsymbol{j}=\rho_{0} \boldsymbol{v}+\boldsymbol{n} \rho_{0} v^{2} / c
\end{gathered}
$$

$\boldsymbol{n}$ is a unit vector in the direction of $\boldsymbol{v}$ and $\partial_{t} E+\nabla \cdot\left(\rho^{\prime} \mathbf{v}\right)=0$. 
When the disturbance propagates in the z-direction, the wave Equation (7) becomes

$$
\partial_{z}^{2} \phi-c^{-2} \partial_{t}^{2} \phi=0
$$

with the plane wave solutions in which $f$ is an arbitrary function and $k^{2}=\omega^{2} / c^{2}$

$$
\phi(z, t)=f(k z-\omega t)
$$

and in particular the harmonic plane waves

$$
\phi(z, t)=\exp [i(k z-\omega t)]
$$

used to perform the Fourier transform of the $f$ functions.

\section{Pulsed Sound Waves}

We now suppose that the functions $\phi(z, t)$ has the form with $k c=\omega$

$$
\phi(u)=A u U(u), u=\sin \tau, \tau=k z-\omega t
$$

in which $U$ is the unit step function and $A$ a constant amplitude with the dimension $[A]=[L]^{2}[T]^{-1}$. Then since $u \delta(u)=0$ where $\delta(u)=d U(u) / d u$ is the Dirac distribution, we get

$$
\begin{gathered}
\partial_{t} \phi=\partial_{u} \phi d u / d t=U d u / d t, \\
d u / d t=d u / d \tau \quad d \tau / d t=\cos \tau d \tau / d t
\end{gathered}
$$

and with the signum function $\operatorname{sgn}(\tau)=1$ for $\tau>0$ and -1 for $\tau<0$

$$
d \tau / d t=-\omega \operatorname{sgn}(\tau)
$$

so that according to (13) and (13a)

$$
\begin{gathered}
\partial_{t} \phi=-\omega \operatorname{sgn}(\tau) \cos \tau U(\sin \tau), \\
\partial_{t}^{2} \phi=\omega^{2}\left[-\sin \tau U(\sin \tau)+\cos ^{2} \tau \delta(\sin \tau)\right]
\end{gathered}
$$

a similar calculation gives

$$
\begin{gathered}
\partial_{z} \phi=k \operatorname{sgn}(\tau) \cos \tau U(\sin \tau), \\
\partial_{z}^{2} \phi=k^{2}\left[-\sin \tau U(\sin \tau)+\cos ^{2} \tau(\sin \tau)\right]
\end{gathered}
$$

It is checked at once that $\phi$ is solution of the wave Equation (10) and taking into account (14) we get from (6) and (8)

$$
\begin{gathered}
p^{\prime}=A \rho_{0} \omega \operatorname{sgn}(\tau) \cos \tau U(\sin \tau) \\
\rho^{\prime}=A c^{-2} \rho_{0} \omega \operatorname{sgn}(\tau) \cos \tau U(\sin \tau) \\
v=A c^{-2} \omega \operatorname{sgn}(\tau) \cos \tau U(\sin \tau)
\end{gathered}
$$

while for travelling waves the energy and momentum densities have the simple form

$$
\begin{gathered}
E=\rho_{0} v^{2}, j_{x}=j_{y}=0, \\
j_{z}=c \rho^{\prime}\left(1+\rho^{\prime} / \rho_{0}\right)
\end{gathered}
$$

We now come to the important property of these disturbances. Since $\sin \tau=0$ for $\tau=n \pi$ where $n$ is an arbitrary integer, the unit function $U(\sin \tau)$ and the Dirac distribution $\delta(\sin \tau)$ have the expansions [5]

$$
\begin{gathered}
U(\sin \tau)=\sum_{n}(-1)^{n} U(\tau-n \pi), \\
\delta(\sin \tau)=\sum_{n} \delta(\tau-n \pi)
\end{gathered}
$$

So, these disturbances are pulsed sound waves made of a sequence of elementary unit step functions.

Remark: The relation $\omega=c k$ is valid for a sound wave propagating in a medium at rest and, in particular, in a frame $K^{\prime}$ relative to a fluid moving with a velocity $V$ so that in the fixed frame $K$ we get the Doppler formula in which $\theta$ is the angle of $\boldsymbol{V}$ with $o z$.

$$
\omega=k c[1-(|\boldsymbol{V}| / c) \cos \theta]
$$

\section{Discussion}

An important question concerns the possibility to generate sound waves of the elementary unit pulse type. Sound waves are produced by oscillating bodies with as prototypes a sphere pulsating in any manner and a cylinder oscillating perpendicularly to its axis [4]. The velocity potential $\phi$, taken as the fundamental quantity, is solution of the wave Equation (7) with the boundary condition $v=$ $V n$ on the surface of the body and it satisfies the Sommerfeld radiation condition at infinity. We follow closely [4] to get the energy of sound waves emitted by oscillating bodies.

We assume here that the body oscillating with a pulsation $\omega$, in the $z$-direction, is a small cube parallel to the coordinate axis with a characteristic dimension 1 so that its volume and the area of its lateral faces are respectively 13 and 12 . Then, $\lambda=2 \pi \omega / c$ being the wave length of the emitted wave, we consider two asymptotic situations $\lambda \ll 1$ and $\lambda \gg 1$ in which the actual shape and the dimensions of the oscillating body do not intervene at distances far from this body. So, we may assume spherical waves as in Sec. 73 of [4].

For $\lambda \ll 1$, the mean energy $I$ emitted per unit time in the form of sound waves is [4]

$$
I=c \rho l^{2}\left\langle V_{z}^{2}\right\rangle
$$

in which $V_{z}$ is the vertical velocity of the $x, y$ planes, (the usual notation: a bar over a quantity to denote its mean value being not available here, we use the symbol $<>$ ).

The situation is more intricate for $\lambda \gg 1$ but at large distances $r \gg \lambda$ where $r$ is the distance from an origin anywhere inside the oscillating body, the velocity potential $\phi$ is a solution of the Laplace equation $\Delta \phi=0$ with the solutions 


$$
\phi=-a / r+\boldsymbol{A} \cdot \nabla(1 / r)
$$

Then, it is shown that the velocity $\boldsymbol{v}=\nabla \phi$ is when the emitting body undergoes pulsations during which its volume $V$ changes

$$
\boldsymbol{v}=\boldsymbol{n} \partial_{t}^{2}(t-r / c) / 4 \pi c \boldsymbol{r}
$$

in which $\boldsymbol{n}$ is a unit vector in the direction of $\boldsymbol{r}$ and $\partial_{t}^{2} V$ the variation of volume.

Then, the mean value of the total energy emitted per unit time is

$$
I=\rho c \int\left\langle v^{2}\right\rangle \mathrm{d}
$$

where the integration is taken on a surface surrounding the origin. So, substituting (20) into (21) and taking as surface a sphere of radius $r$ we have finally

$$
I=\rho\left\langle\left(\partial_{t}^{2} V\right)^{2}\right\rangle / 4 \pi
$$

If the body executes harmonic pulsations of frequency $\omega$, the intensity of emission is proportional to $\omega^{4}$.

The situation is still different for a body oscillating without changes of volume. Then one has to deal with a dipole emission [4] characterized by a dipole vector $\boldsymbol{A}$

$$
\phi=-\partial_{t} \boldsymbol{A}(t-r / c) \cdot \boldsymbol{n} / c
$$

and

$$
\boldsymbol{v}=\boldsymbol{n}\left(\boldsymbol{n} \cdot \partial_{t}^{2} \boldsymbol{A}\right)
$$

so that

$$
I=\rho / c^{3} \int\left\langle\left(\boldsymbol{n} \cdot \partial_{t}^{2} \boldsymbol{A}\right)^{2}\right\rangle / r
$$

Taking the surface of integration to be a sphere of radius $r$ and using spherical coordinates with the polar axis in the direction of the vector $\boldsymbol{A}$, we finally have

$$
I=4 \pi \rho\left\langle\left(\partial_{t}^{2} \boldsymbol{A}\right)^{2}\right\rangle
$$

and, if the body executes harmonic oscillations of frequency $\omega, I$ is proportional to $\omega^{6}$.

Thus from a theoretical viewpoint, it is possible to generate sound waves becoming a sequence of elementary unit pulses at large distances of an oscillating small cube.

Sound waves may also be generated with the help of pulsed electromagnetic beams [6-8] The pulsed sound waves investigated here have simple analytical expressions making them particularly suitable for computer simulation of the processes in which they are involved.

\section{References}

[1] C. Boyd-Brewer, "Vibro Acoustic the Rapy: Sound Vibrations in Medicine," Alternative and Complementary Therapies, Vol. 9, 2005, pp. 257-263.

[2] Sonar Site Internet (Wikipedia.)

[3] Ch. Hellier, "Ultrasonic testing in Hand-book of Nondestructive Evaluation," Mac Graw Hill, New York, 2003.

[4] L. D. Landau and E. M. Lifshitz, "Fluid Mechanics," Pergamon, London, 1959.

[5] B. Van Der Pol and H. Bremmer, "Operational Calculus," University Press, Cambridge, 1959.

[6] B. J. Van Gutfeld and R. L. Melcher, "20 MH Acoustic Waves from Pulsed Thermoelastic Expansion of Constrained Surface," Applied Physics Letters, Vol. 30, No. 6, 1977, pp. 257-259. doi:10.1063/1.89375

[7] A. Puskarev, J. Isakova, G. Kholudnaya and R. Sazonov, "Sound Waves Due to the Absorption of a Pulsed Electronic Beams," Advances in Sound Localization Intech, 2011.

[8] C. Bacon, E. Guillorit, B. Hosten and D. Chimenti, "Acousric Waves by Pulsed Microwaves in Viscoelastic Rods," Journal of the Acoustical Society of America, Vol. 110, 2001, pp. 1396-1407. doi:10.1121/1.1391241 